\title{
Of metals, mice, and men: what animal models can teach us about body iron loading
}

\author{
Gregory J. Anderson and Lawrie W. Powell
}

Joint Clinical Sciences Program, The Queensland Institute of Medical Research and The University of Queensland, PO Royal Brisbane Hospital, Brisbane Queensland, Australia

Address correspondence to: Gregory J. Anderson, Clinical Sciences Unit, Queensland Institute of Medical Research, PO Royal Brisbane Hospital, Brisbane Queensland 4029, Australia. Phone: 61-7-3362-0177;

Fax: 61-7-3362-0191; E-mail: gregA@qimr.edu.au.

Hemochromatosis is a common inherited disease of iron overload that results from mutations in the HFE gene (1). It has long been known that basic processes of body iron homeostasis, and in particular intestinal iron absorption, are regulated aberrantly in hemochromatosis, so an understanding of how HFE functions in cellular and body iron homeostasis may hold the key to dissecting these essential physiological processes. However, despite a growing body of elegant experimentation, the recalcitrant HFE molecule has yet to reveal all its secrets.

The clinical features of hemochromatosis are almost exclusively due to increased iron storage in the parenchymal cells of many tissues, particularly the liver (2). However, the iron accumulation and the associated clinical presentation can be highly variable, even within one family, and this remains one of the most puzzling aspects of the disease. A recent population study (3) found that only half of those who were homozygous for the major HFE mutation had clinical features of hemochromatosis and one quarter had serum ferritin levels (a marker of body iron stores) that remained normal over a 4-year period. Environmental factors such as physiological and pathological blood loss sometimes explain these differences, but often they do not, and it seems more likely that genetic factors are involved. Furthermore, there are clear cases, particularly in Mediterranean populations

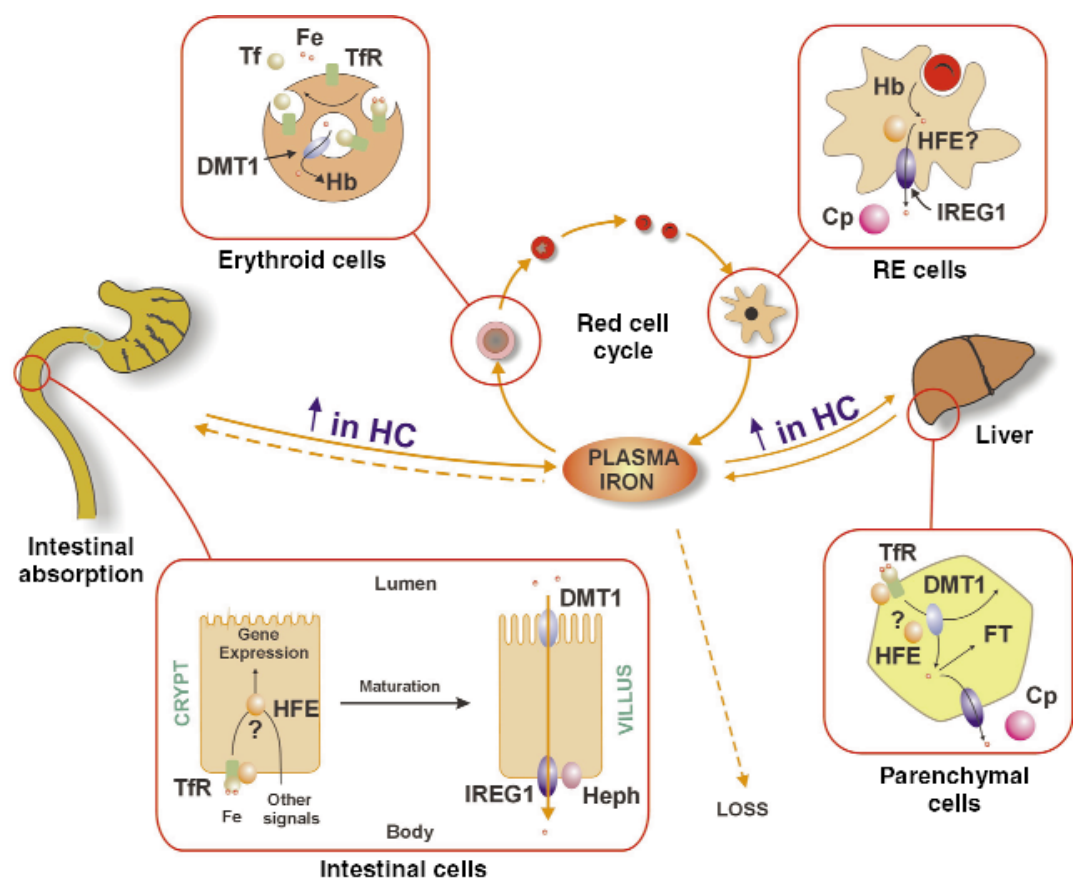

Figure 1

Pathways of iron metabolism showing sites where HFE may play a role. The basic components of iron influx and efflux are shown in intestinal, erythroid, reticuloendothelial (RE), and parenchymal cells. Tf, transferrin; Cp, ceruloplasmin; Fe, iron; Heph, hephaestin; FT, ferritin; $\mathrm{Hb}$, hemoglobin; $\mathrm{HC}$, hemochromatosis.

(4), of iron overload in which HFE coding sequences are normal. Thus, genes other than HFE and/or HFE mutations in introns or in flanking regulatory sequences may be responsible.

One might expect that the gene defective in a disorder with a variable phenotype would contain a spectrum of mutations with varying functional consequences, but this is not the case for hemochromatosis. A single missense mutation in HFE has been found to be responsible for the vast majority of clinical disease, so mutations in other genes must be invoked to explain the differences in iron loading. Three recent clinical studies (5-7) have provided evidence for hemochromatosis-modifying genes, but the identity of these genes is not known. The obvious candidate genes are those encoding proteins known to be important in iron transport. In this issue, Levy et al. (8) have examined the effects of several of these genes on iron loading by crossing HFE knockout mice (which show a phenotype very similar to human hemochromatosis) with other strains of mice with natural or engineered defects in other genes affecting iron homeostasis. While some of the results were predictable, others were not.

A major pathophysiological feature of hemochromatosis is an increase in intestinal iron absorption, so one might predict that mutations in genes important for intestinal iron transit would ameliorate the hemochromatosis phenotype. This is indeed the case, and HFE knockout mice carrying mutations in the gene for the brush border iron transporter DMT1 show reduced levels of hepatic iron accumulation. DMT1 is the major molecule facilitating the uptake of dietary iron by the epithelial cells of the proximal small intestine (9, $10)$, and mice defective in DMT1 ( $m k$ mice) have a profound microcytic, hypochromic anemia. The demonstra- 
tion by Levy and colleagues (8) that deletion of the HFE gene cannot correct the mk phenotype shows that DMT1 is the major route of iron entry into intestinal epithelial cells in hemochromatosis.

Genetic interactions between HFE and DMT1 are only part of the story. The intestinal epithelial cell is a polarized cell and iron must traverse not only the apical membrane but the basolateral membrane as well. Insights into this iron efflux step have come from the cloning of the gene mutated in sla mice (11), another mouse strain with an inherited anemia. The affected gene has been named Hephaestin and it bears significant homology to the copper-containing plasma protein ceruloplasmin, which has long been known to play a role in iron release from tissues. HFE knockout mice carrying the sla mutation show considerably reduced hepatic iron levels, indicating that hephaestin plays an important role in iron loading. However, since the block to absorption is not complete, alternative basolateral iron transfer pathways appear to be operating. We would expect that disruption of the newly identified basolateral iron transporter gene IREG1 (or ferroportin1) $(12,13)$ would also ameliorate the iron overloading phenotype of HFE knockout mice.

Somewhat more unexpected was the finding that HFE knockout mice also lacking the $\beta_{2}$-microglubulin $(\beta 2 m)$ gene showed significantly more iron loading than HFE knockout mice with wild-type $\beta 2 \mathrm{~m}$. Since HFE is a member of the MHC class I family, an interaction with $\beta 2 \mathrm{~m}$ is essential for its correct cellular processing and function, and deletion of the $\beta 2 m$ gene results in an iron overload phenotype (14). However, the findings of Levy et al. (8) suggest that HFE is not the only $\beta 2 \mathrm{~m}$-associated protein that participates in body iron loading and support the evidence that another gene in the distal MHC is involved in this pathway (7).

The most intriguing finding of this study comes from a cross between HFE knockout mice (or mice carrying the major hemochromatosis mutation in their HFE gene) and mice lacking one transferrin receptor $(\operatorname{Trfr}$ ) allele. Mice lacking both Trfr alleles die in utero and cannot be studied, but mice with defects in HFE and with haploinsufficiency for the Trfr show a higher degree of hepatic iron loading than do other animals deficient in HFE. A decreased concentration of transferrin receptors in the erythroid compartment would be expected to promote iron-deficient erythropoiesis, which in turn would stimulate intestinal iron absorption. Decreased iron delivery to the intestinal crypt cells would exacerbate this effect and could lead to increased body iron content. More puzzling, however, is the situation when HFE is normal and one Trfr allele is missing. Animals with this genotype are either mildly iron-deficient (15) or normal with respect to iron status (8), indicating that haploinsufficiency for Trfr leads to increased iron loading only in the presence of defective HFE. The interaction between HFE and Trfr has been well documented (16), but the physiological relevance of this interaction is still unclear. Levy et al. suggest that the two gene products together establish a "set point" for body iron homeostasis, although no mechanism is proposed for how this might affect iron absorption and other iron trafficking within the body.

Are these studies relevant to iron homeostasis in humans? The answer is almost certainly yes, but to what degree variations in the known iron transport genes contribute to the clinical presentation of hemochromatosis and other iron disorders is not known. Several groups have sought mutations in DMT1, $\beta 2 m$, and Trfr in individuals with iron overload, but these small studies have not yet demonstrated a clear association between polymorphisms in these genes and iron loading. The field of iron homeostasis is in a state of rapid flux. The last four years has seen the identification of several important genes of iron transport, and we are only just beginning to understand how they function to control iron uptake and release. Furthermore, it is likely that there are other genes that play important roles in determining iron status that have yet to be described. Under- standing how these molecules operate in vivo is even more challenging. Iron homeostasis results from a complex interplay of signals and responses, and it is difficult to study such a complicated physiological process in vitro or in cell culture models. Consequently, in vivo approaches, such as the present one by Levy and colleagues, hold our best hope for elucidating the causes of human diseases of iron metabolism such as hemochromatosis.

1. Feder, J.N., et al. 1996. A novel MHC class I-like gene is mutated in patients with hereditary haemochromatosis. Nat. Genet. 13:399-408.

2. Powell, L.W., and Yapp, T.R. 2000. Hemochromatosis. Clinics in Liver Disease. 4:211-228.

3. Olynyk, J.K., et al. 1999. A population-based study of the clinical expression of the hemochromatosis gene. N. Engl. J. Med. 341:718-724.

4. Carella, M., et al. 1997. Mutation analysis of the HLA-H gene in Italian hemochromatosis patients. Am. J. Hum. Genet. 60:828-832.

5. Crawford, D.H.G., et al. 1995. Evidence that the ancestral haplotype in Australian hemochromatosis patients may be associated with a common mutation in the gene. Am.J. Hum. Genet. 57:362-367.

6. Whitfield, J.B., et al. 2000. Effects of HFE C282Y and $\mathrm{H} 63 \mathrm{D}$ polymorphisms and polygenic background on iron stores in a large community sample of twins. Am. J. Hum. Genet. 66:1246-1258.

7. Pratiwi, R., et al. 1999. Linkage disequilibrium analysis in Australian haemochromatosis patients indicates bipartite association with clinical expression. J. Hepatol. 31:39-46.

8. Levy, J.E., Montross, L.K., and Andrews, N.C. 2000. Genes that modify the hemochromatosis phenotype in mice. J. Clin. Invest. 105:1209-1216.

9. Fleming, M.D., et al. 1997. Microcytic anaemia mice have a mutation in Nramp2, a candidate iron transporter gene. Nat. Genet. 16:383-386.

10. Gunshin, H., et al. 1997. Clonin and characterization of a mammalian proton-coupled metalion transporter. Nature. 388:482-488.

11. Vulpe, C.D., et al. 1999. Hephaestin, a ceruloplasmin homologue implicated in intestinal iron transport, is defective in the sla mouse. Nat. Genet. 21:195-199.

12. McKie, A.T., et al. 2000. A novel duodenal ironregulated transporter, IREG1, implicated in the basolateral transfer of iron to the circulation. Mol. Cell. 5:299-309.

13. Donovan, A., et al. 2000. Positional cloning of zebrafish ferroportin 1 identifies a conserved vertebrate iron exporter. Nature. 403:776-781.

14. Santos, M., et al. 1996. Defective iron homeostasis in b2-microglobulin knockout mice recapitulates hereditary hemochromatosis in man. J. Exp. Med. 184:1975-1985.

15. Levy, J.E., Jin, O., Fujiwara, Y., Kuo, F., and Andrews, N.C. 1999. Transferrin receptor is necessary for development of erythrocytes and nervous system. Nat. Genet. 21:396-399.

16. Eisenstein, R.S. 1998. Interaction of the hemochromatosis gene product HFE with transferrin receptor modulates cellular iron metabolism. Nutr. Rev. 56:356-358. 